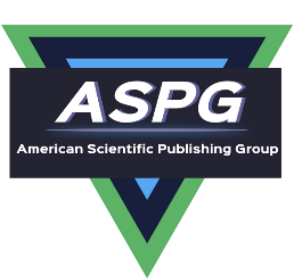

\title{
A Proposed AI-based Algorithm for Safety Detection and Reinforcement of Photovoltaic Steel
}

\author{
Ali A. Alwan1, Abedallah Zaid Abualkishik2** \\ ${ }^{1}$ School of Theoretical \& Applied Science, Ramapo College of New Jersey, USA \\ ${ }^{2}$ American University in the Emirates, Dubai, UAE \\ Email: aaljuboo@ramapo.edu, Abedallah.abualkishik@aue.ae
}

\begin{abstract}
:
In the era of fossil energy depletion and increasing environmental pollution, clean and renewable new energy represented by photovoltaic power generation has become an increasingly important part of multinational companies' energy structure. With the advent of the era of photovoltaic parity, the use of photovoltaic tracking systems has become the best choice for many new large-capacity power stations. The cost of the support occupies a very large proportion in the investment of the entire power station construction. Therefore, the rationality of the design of the support, cost control and service life have become important ways for competition in the photovoltaic support industry. Based on the above background, the research content of this article is the application of artificial intelligence algorithms in the safety detection and reinforcement of photovoltaic steel supports. To be able to pass the monitoring data, this paper applies intelligent algorithms to perform faster and more accurate safety inspections on photovoltaic steel supports while minimizing labor costs, and to strengthen the photovoltaic steel supports, this paper chooses neural networks as the basic algorithm A structural model of a photovoltaic steel support was proposed. Finally, experimental simulations showed that the wavelet neural network reached 93.87\%. Compared with traditional neural networks, wavelet neural networks perform better in fault prediction accuracy, but the speed needs to be improved. The method proposed in this paper has successfully completed the diagnosis of each component of the photovoltaic bracket in the safety inspection of the photovoltaic steel bracket, and meets the immediateness and accuracy required for the safety inspection of the photovoltaic bracket.
\end{abstract}

Key words: Photovoltaic System, Tracket Reinforcement, Artificial Intelligence Algorithm, Safety Detection, Wind Load

\section{Introduction}

With the third rapid development of artificial intelligence, the application scenarios and service models of intelligent public service robots are continuously expanding, driving the rapid growth of the service robot market. The increasing demand for robots and the development of related technologies have made robots more and more intelligent. 
The photovoltaic industry is one of the fastest-growing emerging industries in the world that uses solar energy for photoelectric conversion technology [1]. It is a technology that directly converts solar radiant energy into electrical energy using the photovoltaic effect principle of semiconductor materials. 3]. Using a solar cell combination square array, a photovoltaic power station can be built, which can provide reliable energy for industry, agriculture, communications, and human life like a conventional power station [4-5]. The solar photovoltaic system mainly includes six parts: the production of polysilicon raw materials, the production of solar cells and modules, the design and processing of photovoltaic brackets, the manufacture of related production equipment, and the construction of photovoltaic power stations [6-7]. Photovoltaic brackets are the core components of solar cell square matrix support structures, and their performance often determines the safe and efficient operation of photovoltaic systems and cost control [89].

Brushless DC motors (BDCMs) are internal self-commutating motors that operate on DC power in industrial and commercial applications. Traditional external BDCM sensors such as Hall-effect and optical encoders are too fragile, expensive, and complex for some applications. Therefore, Khoo uses two types of sensorless BDCM speed estimation techniques: back-EMF (BEMF) and ripple current sensing. The ripple current sensing method provides better accuracy by providing fixed discrete pulses for counting. However, it is covered by noise that is difficult to filter. There are many ways to convert ripple current into pulses: comparator filtering, adaptive filtering, predictive sensing, and pattern recognition. These methods only emphasize the operation of the brake control module when it is close to the operating speed, and ignore other common brake control module operation modes such as braking and coasting. Khoo's research will solve all the problems that are critical in practical applications. The precise position and speed in all modes will affect the precision of the system [10-11]. Fundus images play an important role in the diagnosis of ophthalmic diseases. Most eye diseases manifest on the retina. Kuppusamy proposed a method to detect glaucoma disease by segmenting the optic disc and blood vessels using edge and gradient horizontal segmentation algorithms. Threshold segmentation was performed on the image using the BAT algorithm. Artificial neural network (ANN) is used for glaucoma detection, which involves the training and detection process of glaucoma. Application of support vector machine (SVM) to classify the approximate stage of glaucoma. By comparing the features of the normal image and the extracted image area, the optic disc and blood vessels were accurately identified and measured. The area of glaucoma is measured and thus the stage of glaucoma is provided. The accuracy rate is 98\% [12-13]. Artificial intelligence aims to simulate human cognitive functions. Jiang investigated the current status of artificial intelligence applications in the medical field. Artificial intelligence can be applied to various types of medical data. Popular artificial intelligence technologies include machine learning methods for structured data, and major disease areas that use artificial intelligence tools include cancer, neurology, and cardiology. Then Jiang reviewed in detail the application of artificial intelligence in the three major aspects of early stroke detection and diagnosis, treatment, prognosis prediction and prognosis evaluation. Finally, Jiang discussed pioneering artificial intelligence systems such as IBM Watson and the obstacles to the deployment of artificial intelligence in real life [14-15]. The demand for higher-quality global medical services is growing, and the development of computer- and robot-based medical tools that rely on artificial intelligence technology has expanded accordingly. The Turing test is designed to evaluate artificial intelligence (AI) in computer technology. It remains an important qualitative tool for testing the next generation of medical diagnostics and medical robots. Ashrafian has improved the Turing test. Ashrafian provides quantifiable diagnostic accuracy and statistical effect-size robustness when evaluating artificial intelligence in computer- and robot-based

DOI: $10.5281 /$ zenodo.5203176

Received: January 16, $2021 \quad$ Accepted: May 13, 2021 
medical technology. Ashrafian's improved Turing test artificial intelligence provides reliable diagnostic scores, helping to improve and improve next-generation digital diagnostics and medical robotics [16-17].

This paper explores the working mechanism of photovoltaic cells and modules and sets up equivalent circuit models and mathematical models of photovoltaic cells, and analyzes and compares the factors affecting the maximum output power of photovoltaic modules. The artificial intelligence algorithm and wavelet neural network are used to strengthen and secure the photovoltaic bracket. Finally, a finite element model of the photovoltaic bracket system device is established in the finite element ANSYS software, and static analysis and modal analysis are performed for different working conditions. The structure of the photovoltaic support system is subjected to a static load test to verify the rationality and reliability of the theoretical calculation results and the finite element analysis results.

\section{Proposed Method}

\subsection{Artificial Intelligence Algorithm}

Artificial intelligence is a new technological science researched and developed on the basis of computer science (CS), which is used to simulate and expand human intelligent behavior [18-19]. There are four major branches of artificial intelligence technology, including machine learning, data mining, pattern recognition, and intelligent algorithms. Machine learning is an interdisciplinary [20-21]. Because of its wide application, machine learning has different definitions: it belongs to a branch of artificial intelligence and optimizes the performance of specific algorithms through empirical learning; it is the study of computer algorithms that can automatically improve learning through experience; it uses data or Past experience, automatic analysis to obtain the rules, in order to optimize the performance criteria of computer programs [22-23]. Machine learning is mainly divided into the following categories: the training set of supervised learning requires features and goals, where the goals are artificially labeled; however, the training set of unsupervised learning is not artificially labeled; between The algorithm is semi-supervised learning; reinforcement learning evaluates whether the feedback after the action is positive or negative [24-25].

The model fusion technology that generates new models from the information of multiple prediction models is called meta combination (also called stacking) [26-27]. In general, the principle of stacked models is to increase the proportion of basic models that perform well, and to reduce the proportion of basic models that perform poorly [28]. Therefore, the effect of the stacked model will be better than that of each single basic model, that is, when there are significant differences in the basic model, the stacked model is the most effective [29-30]. Model fusion is a kind of stacked model and a powerful technique to improve accuracy. The principle of model fusion is to improve the accuracy of the model by training multiple models and integrating the multiple models according to a certain method. Because it is easy to understand and simple to implement, and also has good results, there are three common strategies for model fusion: average method, voting method, and learning method. Among them, the average method is mostly used for regression prediction models, the voting method is often used for classification models, and the learning rule is a strong combination strategy.

Logistic regression model is a classic discrimination method to solve classification problems. The classification problem can be analyzed from multiple angles. The binomial logistic regression model is represented by a conditional probability distribution $\mathrm{P}(\mathrm{Yx})$, where the range of the random variable $\mathrm{Y}$ is 1 
or 0 . When 1 is taken, it indicates a positive sample, and when 0 is taken. Represents a negative sample; the range of the random variable $X$ is a real number. The binomial logistic regression model is

$$
\begin{aligned}
& P(Y=1 \mid x)=\frac{\exp (W \cdot x)}{1+\exp (W \cdot x)} \\
& P(Y=0 \mid x)=\frac{1}{1+\exp (W \cdot x)}
\end{aligned}
$$

Suppose there are $\mathrm{N}$ samples, and each sample $\mathrm{f}$ is composed of independent variable $\mathrm{x}_{\mathrm{i}}$ and corresponding dependent variable $Y_{i}$. When $Y_{i}=1$, it represents a positive sample, and when $Y_{i}=0$, it represents a negative sample. Assume that $\mathrm{y}$. Is independent and obeys Bernoulli distribution, which is:

$$
\begin{gathered}
P(Y=1 \mid x)=\pi(x) \\
P(Y=0 \mid x)=1-\pi(x)
\end{gathered}
$$

The likelihood function is

$$
\prod_{i=1}^{N}\left[\pi\left(x_{i}\right)\right]^{y_{i}}\left[1-\pi\left(x_{i}\right)\right]^{1-y_{i}}
$$

When formula (1) and formula (2) are brought into formula (5), the log-likelihood function formula can be

$$
\begin{gathered}
L(W)=\sum_{i=1}^{N}\left[y_{i} \log \pi\left(x_{i}\right)+\left(1-y_{i}\right) \log \left(1-\pi\left(x_{i}\right)\right)\right] \\
L(W)=\sum_{i=1}^{N}\left[y_{i} \log \left(\pi\left(x_{i}\right) / 1-\pi\left(x_{i}\right)\right) \log \left(1-\pi\left(x_{i}\right)\right)\right] \\
L(W)=\sum_{i=1}^{N}\left[y_{i}\left(W \cdot x_{i}\right)-\log \left(1+\exp \left(W \cdot x_{i}\right)\right)\right]
\end{gathered}
$$

By using the maximum likelihood estimation method to find the extreme value of the above formula, the estimated value of $\mathrm{W}$ can be obtained. Therefore, this problem becomes an optimization problem with $\mathrm{L}(\mathrm{W})$ as the objective function, which is usually solved by gradient descent method and quasi-Newton method. The multinomial logistic regression model is

$$
\begin{gathered}
P(Y=k \mid x)=\frac{\exp \left(W_{k} \cdot x\right)}{1+\sum_{k=1}^{K-1} \exp \left(W_{k} \cdot x\right)}, k=1,2, \ldots, K-1 \\
P(Y=k \mid x)=\frac{1}{1+\sum_{k=1}^{K-1} \exp \left(W_{k} \cdot x\right)}
\end{gathered}
$$


The parameter estimation principle of the multinomial logistic regression model is the same as that of the binomial logistic regression model. The multi-layer perceptron algorithm is shown in Figure 1.

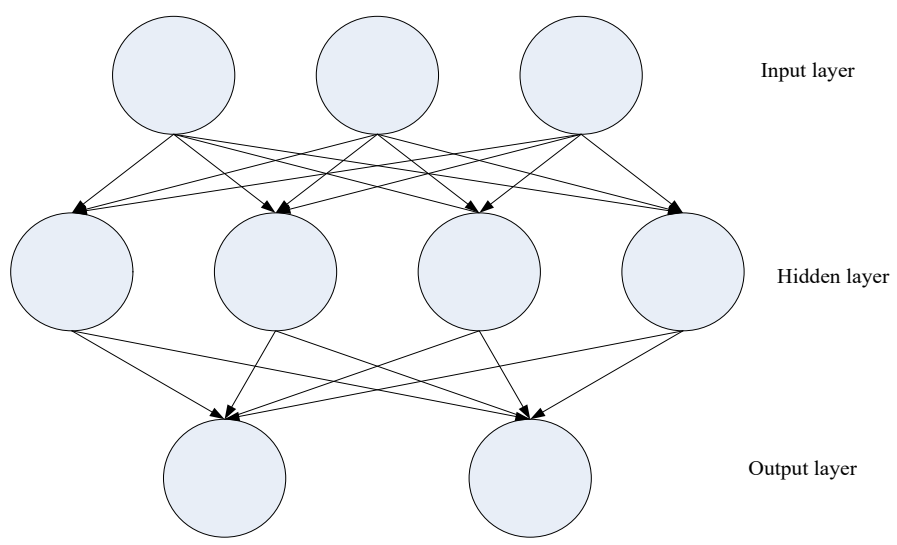

Figure 1. Multilayer Perceptron Algorithm

From the hidden layer to the output layer, it can usually be regarded as a multi-class logistic regression model, that is, soflmax regression MLP is a typical deep learning model, which can solve more complex problems. Compared with machine learning models, MLP has many characteristics. First, in theory, it can approximate all non-linear mappings; second, it has a high degree of connectivity; finally, it has strong selfadaptation and self-learning capabilities, which is helpful to improve the accuracy of prediction. However, because it uses hidden layers, it makes the learning process extremely difficult.

Stacking is a more popular model fusion method. Generally, a high-level basic learner is combined with a lower-level learner to obtain higher prediction accuracy. Although Stacking was proposed many years ago, it is less used than Bagging and Boosting due to its difficult theoretical analysis. Unlike Bagging and Boosting, Stacking is usually used for basic learners composed of different learning algorithms. The algorithm of tacking model fusion is shown in Figure 2.

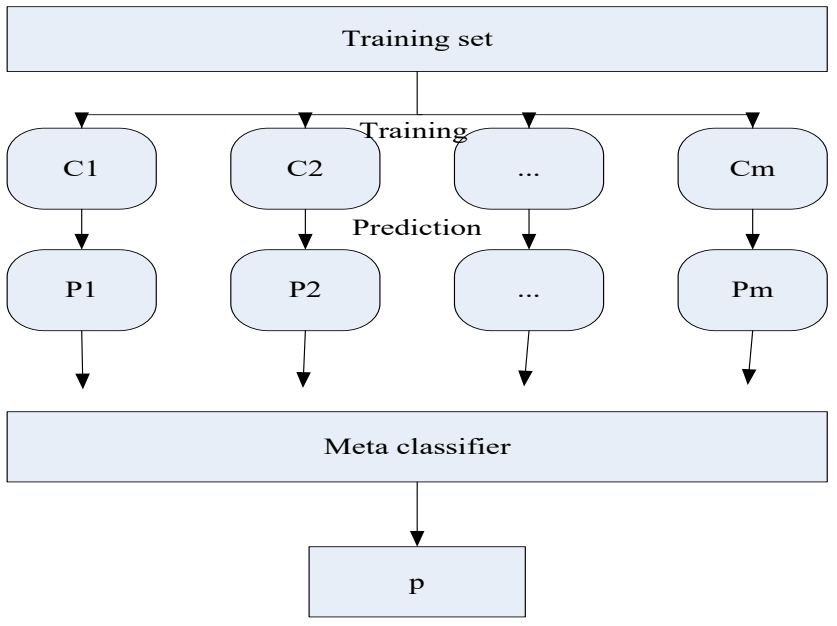

Figure 2. Stacking model fusion

Generally, the Stacking model is divided into two phases. The first stage trains multiple different models, and then uses the model results obtained in the first stage as the input of the second stage model 
to obtain the final prediction results. The algorithmic idea of the Stacking model is: in the first stage, multiple different models (different types of models or models with different parameters) are used to fit the training set respectively.

\subsection{Photovoltaic Steel Bracket}

The bracket device is one of the important factors to ensure the normal operation of the photovoltaic system and affect its life. Three types of tracking photovoltaic bracket systems currently widely used are: horizontal single-axis tracking bracket, oblique single-axis tracking bracket and dual-axis tracking bracket. From the perspective of construction convenience and input cost, domestic horizontal single-axis tracking brackets are used more. The main shaft is horizontally installed. The solar panel can rotate along with the main shaft, and the main shaft is rotated by the sensor tracking the sunlight. The flat uniaxial tracking bracket is usually a support form of a main beam, which supports the solar panel through a single main beam, and completes the task of tracking rotation. This allows the main beam of the bracket to support two bending and torsion states at the same time, which increases the burden on the bracket, and the central part of the solar panel is supported on a single main beam. When a strong external force is applied, the panel is unevenly stressed, and it is easy to produce partial Excessive stress causes damage to photovoltaic cell modules such as cracking, which ultimately leads to failure to generate electricity normally. Therefore, this chapter designs a double main beam flat uniaxial photovoltaic support structure, focusing on solving the problem of uneven stress loading of solar panels, partial stress on the module and damage, increasing the service life of the equipment and reducing the actual engineering problems of later maintenance costs. Changing the traditional main beam as a support and bearing the state of bending and torsion, the selflubricating material of the main beam of the bracket is prone to wear and damage; the design requirements are reasonable and simple structure, convenient manufacturing and construction, and low cost for popularization and application.

At present, there are several types of bracket materials: (1) carbon steel Q235, the surface is galvanized, which has good corrosion resistance and is widely used; (2) AL aluminum alloy AL6005-T5, which has surface oxidation, light weight, high strength, and good corrosion resistance, Wide range of applications; (3) New fiber composite materials are lightweight, good in strength, good in stress, and low in cost. However, due to long-term high temperature resistance and environmental impact, it is easy to cause shortcomings such as performance aging and poor impact resistance. (4) 304 stainless steel has good anticorrosion performance, but the cost is too high. It is basically not used. It is mainly used for bolts and other accessories. Currently, the selection of bracket materials is mainly concentrated in two categories: (1) and (2). , Rigid deformation, corrosion resistance and other aspects of carbon steel Q235 and aluminum alloy AL6005-T5 comparative analysis.

Table 1. Material performance comparison

\begin{tabular}{lllllll}
\hline $\begin{array}{l}\text { Material } \\
<6 \mathrm{~mm}\end{array}$ & $\begin{array}{l}\text { Anti-stress } \\
\mathrm{N} / \mathrm{mm}^{2}\end{array}$ & $\begin{array}{l}\text { Tensile } \\
\mathrm{N} / \mathrm{mm}^{2}\end{array}$ & $\begin{array}{l}\text { Bending } \\
\text { resistance } \\
\mathrm{N} / \mathrm{mm}^{2}\end{array}$ & $\begin{array}{l}\text { Shear } \\
\text { resistant } \\
\mathrm{N} / \mathrm{mm}^{2}\end{array}$ & $\begin{array}{l}\text { Elastic } \\
\text { Modulus } \\
\mathrm{GPa}\end{array}$ & $\begin{array}{l}\text { Density } \\
\mathrm{g} / \mathrm{cm}^{3}\end{array}$ \\
\hline Q235 & 216 & 216 & 216 & 126 & 205 & 7.86 \\
AL6005-T5 & 216 & 216 & 216 & 126 & 72 & 2.72 \\
\hline
\end{tabular}

As can be seen from Table 1, in terms of material strength, the mechanical properties of carbon steel Q235 are almost equivalent to AL aluminum alloy AL6005-T5. In terms of deflection, the deformation of 
AL6005-T5 is 2.9 times that of Q235, but the weight is only one third of that of carbon steel. In terms of corrosion resistance, the aluminum alloy bracket adopts anodizing process to form a dense oxide film, which has good corrosion resistance. The carbon steel bracket adopts spray painting and hot-dip galvanizing technology, which has good anti-corrosion effect, but has large pollution and high cost. Painting effect is poor, and later maintenance costs are high. In terms of cross-section processing, carbon steel sections mainly include C-shaped steel, Z-shaped steel and round pipes, etc., which are achieved by rolling, bending and punching. Aluminum alloy profiles can be easily processed into any cross-section.

\subsection{Support Safety Inspection and Reinforcement}

In the design and calculation of the reinforced support, the bearing capacity calculation is mostly based on the plane frame calculation theory. This method is simple and easy to apply. However, the nonstructural components such as pipes in the support system also play a certain role in sharing the load in practice. The reinforced support is a composite structure, and its bearing capacity should be analyzed first. When carrying out calculation and analysis of the bearing capacity of the reinforced bracket, the bracket is calculated as a single-piece bracket. The calculation of the single-piece bracket assumes that the beamcolumn connection is assumed to be rigid, and the foundation-column connection is fixed. In the calculation of the internal force of the reinforced bracket, a single-piece plane frame calculation model is used in the plane, and the lower end is fixed outside the plane, and the free bending member at the upper end is subjected to a force analysis. The reinforced support beam is fixed at both ends to calculate the out-of-plane torque of the component under horizontal thrust, and the out-of-plane bending moment is calculated and analyzed based on the simple support at both ends. The temperature load and the elasticity of the compensator at both ends are ignored in the calculation. The longitudinal horizontal load is calculated after the friction of the pipeline and the displacement and rebound of the reinforced pipeline are applied according to the difference of each movable pipeline support. In order to avoid errors caused by other factors in the comparative analysis, the same calculation software as the overall calculation model is used here.The calculated load of the steel-clad reinforced support not only needs to consider the original design load, but also the design weight of the angle steel and flat steel hoop used for reinforcement, as well as the weight of the cement mortar protective layer on the surface of the reinforced welded steel. The internal force is calculated according to the simplified diagram of the unreinforced pipe frame. In the design of the original support structure, the standard value was used as the basic representative value of the load. The load was provided by the corresponding process designer and considered according to the standard value of the load. For the wind load in the plane of the reinforced support, the two parts of the support are calculated separately. The calculated wind load of the support ignores the influence of the reinforcement of the package on the section. Due to the different diameters, the interaction between different pipes and the upper and lower floors must be considered when calculating their wind loads.

In the structural safety test, since the load and the load effect generally have a linear relationship, and the ratio of the two is a constant, the statistical characteristics of the load can be applied to the load effect accordingly when the structural reliability analysis is performed. Except for permanent loads, they are generally variable loads that change with time. It is more practical to describe them with a stochastic process probability model. And for the material properties of structural members, there are further considerations of the differences between the actual material properties and the standard test piece material properties, and the differences between the actual working conditions and the standard test conditions. The statistical analysis of the adversarial force in the reliability evaluation also needs to consider the

DOI: $10.5281 /$ zenodo.5203176

Received: January 16, $2021 \quad$ Accepted: May 13, 2021 
accuracy of geometric parameters and calculation modes. The uncertainty of the geometric parameters of structural members mainly refers to the manufacturing dimension deviation and installation error, etc. Generally, the members can only consider the variations of width, effective height and moment of inertia. The inaccuracy of the basic assumptions and calculation formulas used in the calculation of resistance will cause the uncertainty of the calculation model. Generally, it can be considered by comparing and analyzing with the experimental structure results or more accurate model calculation results.For the bracket, there is no special provision for its load statistical parameters in the relevant specifications, that is, the statistical parameters of general structural loads are used.The ratio of each load effect will affect the reliability of the structural members of the bracket, mainly relative values, not absolute values. value. Another parameter that affects the reliability of a structure is the coefficient of structural importance.It can be known from the structural component bearing capacity design expression and the function function of the component obtained from this expression that the structural safety level and the structural importance coefficient determined by the structural component Reliability has a more important impact.

\section{Experiments}

\subsection{Subjects and Data}

This paper selects a small photovoltaic tracking power station in an ecological park in Jiangyin City, Jiangsu Province. Among them, four independent tracking systems arranged adjacent to each other form a single-axis tracking array. The effects of undulations and construction technology are slightly different after the precise measurement of the support parameters. For the convenience of calculation, the components on three inclined single-axis systems are regarded as an arrangement of $2 * 7$. The coordinate system was established by taking the junction between the south pillar and the ground on the westmost No. 1 bracket as the origin, and the array bracket parameters are shown in Table 2.

Table 2. Ecological garden array four system support parameters

\begin{tabular}{|l|l|l|l|l|l|l|l|}
\hline Numbering & $\beta$ & $\Phi$ & $X$ & $Z$ & $R$ & L & H \\
\hline 1 & 0 & -26 & 0 & 0 & 1.96 & 12 & 3 \\
\hline 2 & 24 & -19 & 8.5 & 0.4 & 1.96 & 8 & 1.2 \\
\hline 3 & 17 & -14 & 17.4 & 0.2 & 1.96 & 8 & 1.3 \\
\hline 4 & 22 & -17 & 25.1 & 0.4 & 1.96 & 8 & 1.2 \\
\hline
\end{tabular}

The units of $\beta$ and $\Phi$ are degrees, and the units of $X, Z, R, L, H$ are meters.

\subsection{Experimental Environment and Settings}

In this paper, the finite element method is used for experimental simulation. The finite element method is a numerical calculation method. Its basic idea is to discretize a continuous solution domain into a combination of free and finite multiple elements connected only at nodes. Use the approximate function hypothesized in each unit to represent the unknown field function to be solved in the solution domain in pieces. The approximate function is usually expressed by the interpolation function of the unknown field function and its derivative at each node of the unit, so that a continuous The infinite degree of freedom problem becomes a discrete finite degree of freedom problem. Because of its strong adaptability, versatility, and good calculation efficiency and accuracy, the finite element method has become the most important tool for mechanical analysis of complex engineering structures.

This paper uses ANSYS software for analysis. ANSYS software is a large-scale general-purpose finite 
element analysis software developed by American ANYSYS Corporation in the early 1970s, which integrates structure, fluid, electric field, magnetic field, and sound field analysis. Can perform various linear and nonlinear static and dynamic analysis of structures; can perform steady-state or transient fluid dynamic analysis; its characteristics and advantages are coupled field analysis, and it is used in aerospace, energy, automotive, manufacturing and other industries Wide range of applications.

\section{Discussion}

\subsection{Security analysis}

Use the existing data to perform photovoltaic safety detection. The method used and the particle swarm algorithm are used to optimize the wavelet neural network. Eight input features are selected as mean, effective value, standard deviation, kurtosis index, waveform index, peak index, and margin index. Pulse indicator. The above 8 indicators can be calculated based on the existing vibration data. When dividing the data, different time, different locations and different working conditions must be considered. According to the above method, the accuracy of the training group and the test group can be obtained, and the trained model can be used to predict the upcoming failures. But the parameters of different models of fan models may be different, we can fine-tune the corresponding parameters. The processed vibration signal was converted into eight indicators such as effective value and standard deviation, and parameters were obtained through training samples, and then tested by test samples. The sample data is shown in Table 3.

Table 3. Sample data

\begin{tabular}{lll}
\hline Part number & Mean & Effective value \\
\hline 1 & 0.1027 & 0.3204 \\
2 & 0.1819 & 0.4265 \\
3 & 0.1893 & 0.4347 \\
4 & 0.1752 & 0.4188 \\
5 & 0.1635 & 0.4048 \\
6 & 0.1232 & 0.3513 \\
7 & 0.1213 & 0.3476 \\
8 & 0.1573 & 0.3963 \\
\hline
\end{tabular}

The experimental simulation results of traditional neural network, wavelet neural network, genetic algorithm optimized wavelet neural network, and particle swarm optimized wavelet neural network are shown in Figure 3.

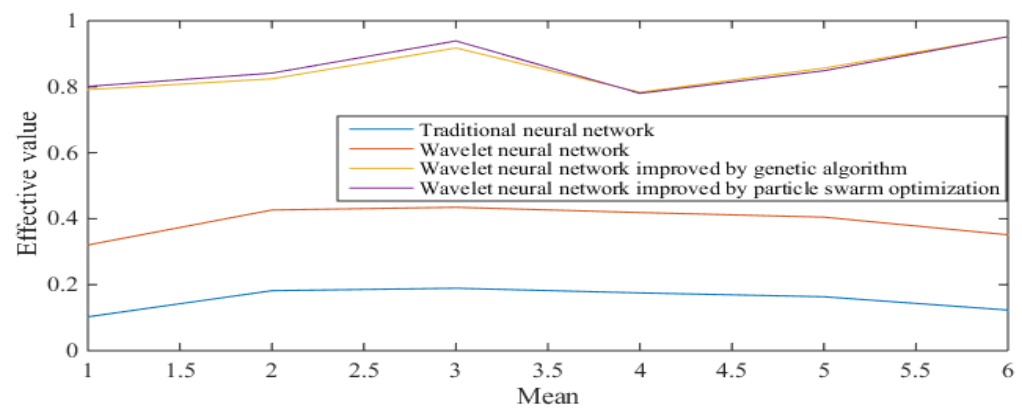

Figure 3. Convergence speed and accuracy 
Combining Table 3 and Figure 3, from the results of simulation predictions, the accuracy of traditional neural networks reached $91.77 \%$, while wavelet neural networks reached $93.87 \%$. Compared with traditional neural networks, wavelet neural networks perform better in fault prediction accuracy, but the speed needs to be improved. The accuracy of the wavelet neural network optimized by the genetic algorithm has reached $95.23 \%$, and the highest accuracy rate has been achieved in the applied algorithm.The shortcoming of the insufficient training speed of the wavelet neural network has been improved to a certain extent, and the prediction has been improved again. Accuracy. The wavelet neural network improved by particle swarm is comparable to the wavelet neural network improved by genetic algorithm in accuracy, reaching an accuracy of $95.16 \%$. But wavelet neural network with particle swarm optimization has obvious advantages in speed. The particle swarm wavelet neural network has successfully completed the diagnosis of the various components of the photovoltaic support in the safety detection of the photovoltaic steel support, which meets the immediacy and accuracy required for the safe detection of the photovoltaic support.

\subsection{Photovoltaic Module Distribution}

The tracking control of photovoltaic modules is generally a set of adjusting mechanisms to control the movement of multiple photovoltaic panels.Since the dimensions of photovoltaic modules are standard, the cross section of the main beam of the bracket designed in this paper is a 80x80 square tube with a thickness of $8 \mathrm{~mm}$. The length is determined by the number of photovoltaic modules it carries. Table 4 shows the size of the main beam for different numbers of modules.

Table 4. Girder sizes with different number of components.

\begin{tabular}{|c|c|c|c|c|c|}
\hline Parameter & 3 components & 4 components & \multicolumn{3}{|c|}{5 components } \\
\hline Contion & Outer $\quad$ outline & square: & 80 & $x$ & 80 \\
\hline section size (mm) & \multicolumn{5}{|c|}{ Internal outline (hollow square): $64 \times 64$} \\
\hline Length (mm) & 4935 & 6555 & & & \\
\hline
\end{tabular}

Under the wind speed of $38 \mathrm{~m} / \mathrm{s}$, the material of the photovoltaic support main beam is Q235 and aluminum alloy AL6005-T5, respectively. The finite element load calculation analysis of the three arrangements is performed.

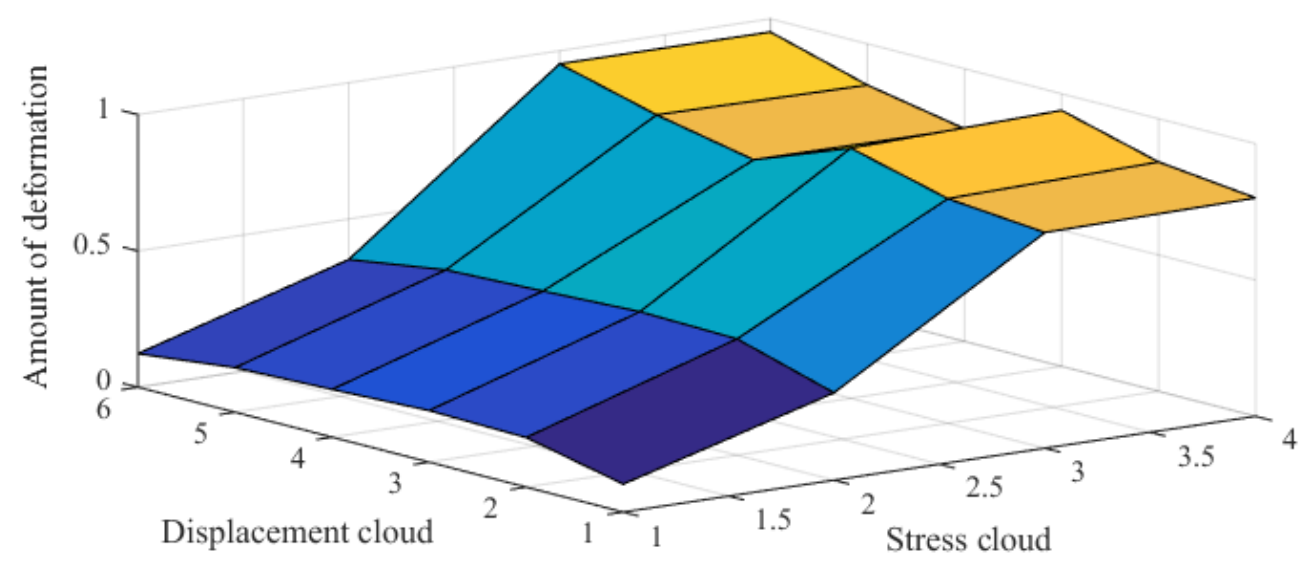

Figure 4. Stress of photovoltaic modules supported by double main beams 
As shown in Figure 4, the overall design requirements and guidelines require that the strength design requirements and stiffness design requirements be met to avoid uneven forces at the joints between the beams and inclined beams of the support system, resulting in local deformation, tearing, and other damage; ensuring that tracking The mechanism parts must not interfere with the movement of the support system. The photovoltaic support structure cannot be deformed too much, otherwise the support device cannot complete the tracking and rotation task, and the solar cell panel may be cracked at the same time, resulting in the failure of normal power generation work. When the double main beam is supported in the form of a group of 5 blocks, although the stress can meet the conditions, the main beam has a large amount of deformation, the deflection has exceeded the allowable amount of deformation, and the stiffness does not meet the requirements. In order to obtain greater structural strength and increase the arrangement density of photovoltaic panels, it is proposed to choose an arrangement of 4 module supports. The structure can not only meet the deflection requirements, but also ensure that the brackets are supported at wind speeds below $38 \mathrm{~m} / \mathrm{s}$. And the components are in a safe state and can meet the requirements of stiffness design.

\subsection{Spectrum Analysis of Pulsating Wind Load}

Natural wind is caused by the uneven pressure of the air in the atmosphere caused by conditions such as heat, resulting in a pressure difference between two points at the same height. Natural wind can generally be decomposed into average wind and pulsating wind. The average wind period is generally more than 10 minutes, while the pulsating wind period is generally only a few seconds to several tens of seconds. The period of the average wind is generally much longer than the natural vibration period of the bracket, so the average wind can be calculated as a static load. The pulsating wind is caused by the irregularity of the natural wind. Generally, the pulsating wind speed at any point in space can be considered as a Gaussian process with various states, and its characteristics can be determined by the power spectral density function. According to the Davenport wind speed spectrum generation method, the wind power spectrum curve of the selected application area for the support area of the project implementation area is shown in Figure 5.

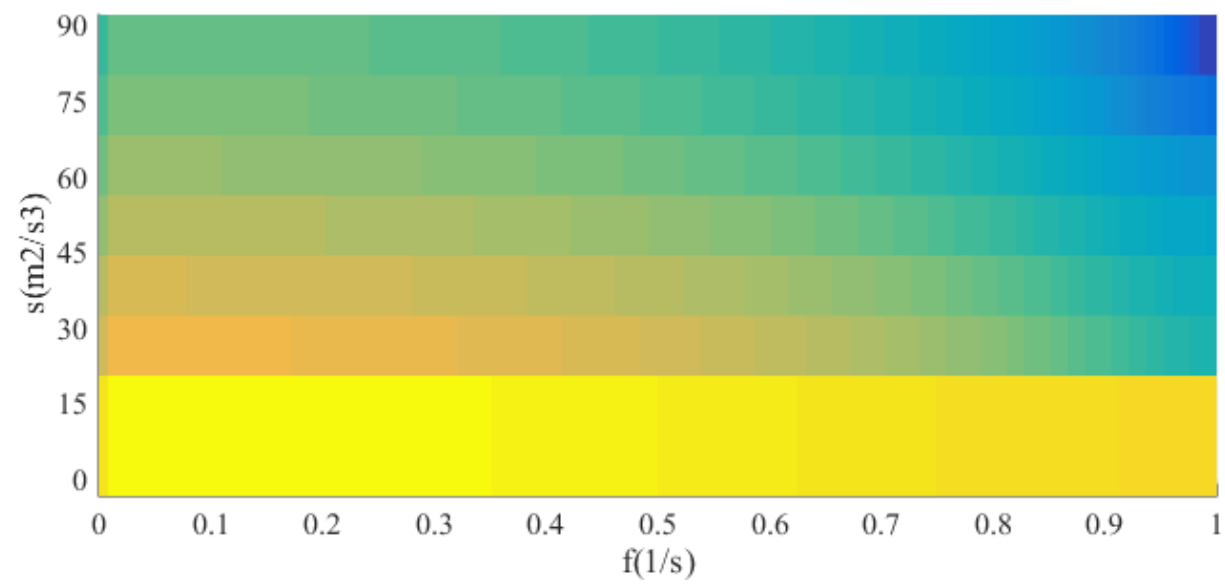

Figure 5. Davenport Power Spectrum Curve

Among them, according to the related research of the building solar photovoltaic system design specification (GB50009-2012), the selected parameters are ground roughness level A and ground roughness coefficient $\mathrm{K}=0.0012910$; $\mathrm{v} 10$ selects the average wind speed at a height of $10 \mathrm{~m}$ at the construction site, The 
design specification (GB50797-2012) and Table E.1 in Appendix E of B / T3811-2008 show that the average wind speed corresponding to the average instantaneous wind speed of $38 \mathrm{~m} / \mathrm{s}$ at $3 \mathrm{~s}$ is $28.88 \mathrm{~m} / \mathrm{s}$. The Davenport power spectrum curve of the photovoltaic bracket designed in this paper decreases rapidly with increasing frequency, and reaches a maximum value when $\mathrm{f}=0.024 \mathrm{~Hz}$, and the maximum value is $3338.67 \mathrm{~ms}$. The energy of the pulsating wind load is mainly concentrated in the low frequency part, so when simulating the pulsating wind load, the high frequency part that accounts for a small proportion of energy, that is, the spectral density after the frequency is greater than $1 \mathrm{~Hz}$ can be ignored.

\subsection{Static Load Analysis of Photovoltaic Support}

The implementation area of the photovoltaic support system belongs to the plateau desert climate environment, accompanied by strong winds.The support system will bear the wind load in the forward and reverse directions alternately, and the front and back surfaces will oscillate and shake, which will accelerate material fatigue, which may cause photovoltaic panels and other The failure of related components makes it difficult to ensure the service life of the photovoltaic support system. Therefore, before mass production and use, it is necessary to perform simulation experiments on mechanical load conditions according to existing standards, and to more objectively and comprehensively verify and evaluate whether the photovoltaic bracket system can withstand static loads and meet the requirements of various working conditions. Only after the test results under the static mechanical load are qualified can they be used in mass production. The allowable and measured deformations of longitudinal beams and transverse beams are shown in Figure 6.

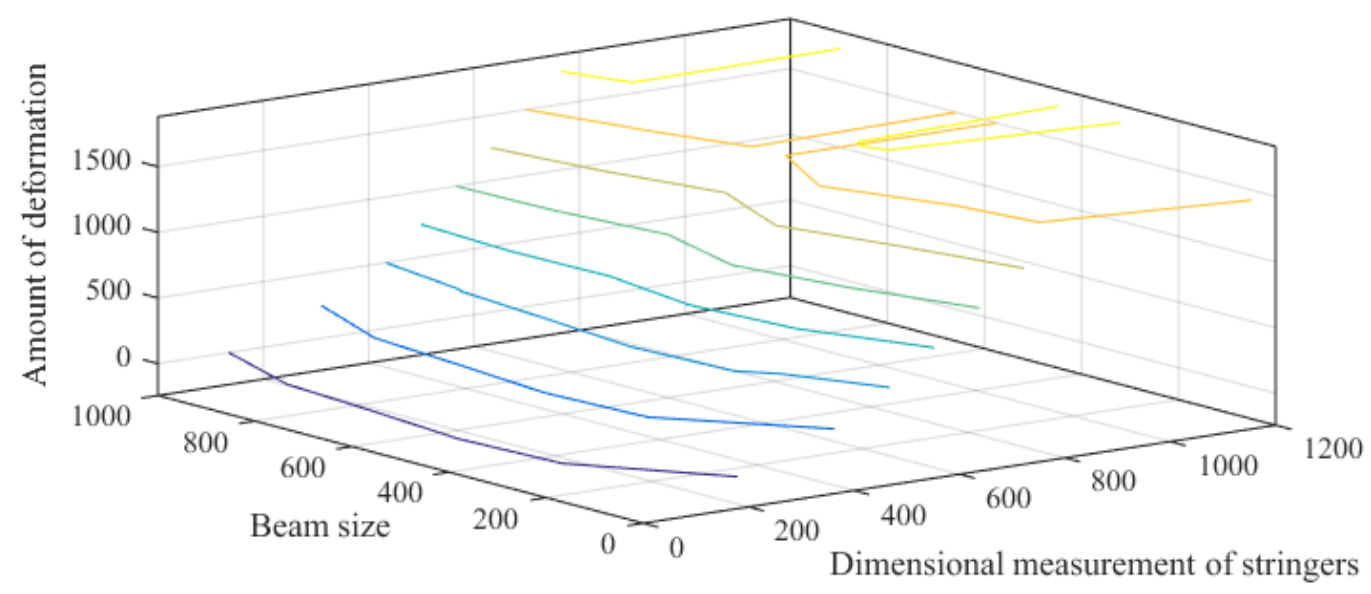

Figure 6. Allowable and measured deformation of longitudinal beams and beams

As shown in Figure 6, the experimental structure is symmetrical. Under static load, the deformation of the beam or longitudinal beam should be consistent. However, because the load is distributed unevenly through the sandbag loading in this experiment, the data in the table are different. The average actual deformation of the beam is $30 \%$ of the allowable deformation; it indicates that the stiffness of the longitudinal beam is good; the average actual deformation of the beam is $75 . \%$ of the allowable deformation; through comparison of the calculation results, it can be seen that the entire structure design, The beam is still a weak point; the test data of the main beam (transverse beam) and longitudinal beam meet the allowable deformation under the load condition, and the design has a safety margin. 


\section{Conclusions}

In this paper, aiming at the problems of uneven stress load, large stress deformation of the existing photovoltaic cell panels, large damage to the panels, and high maintenance costs in the later stage, an artificial intelligence algorithm was used to design a dual main beam flat uniaxial photovoltaic bracket system device. The rigidity of the beam can reduce the photovoltaic module vibration under wind load, which is conducive to improving the efficiency of solar installation and replacement. The computational fluid dynamics software CFD is used to simulate the wind load of the photovoltaic module, and the selection of the project implementation point is determined. rationality.

In this paper, the vibration signal data of the photovoltaic steel bracket is used as the research object. After the vibration signal is preprocessed, different intelligent algorithms are trained, and simulation simulation is performed to predict and compare the different intelligent algorithms for the safety detection of the photovoltaic steel bracket. Effectiveness. From the results of simulation predictions, the accuracy of traditional neural networks reached $91.77 \%$, while wavelet neural networks reached $93.87 \%$. Compared with traditional neural networks, wavelet neural networks perform better in fault prediction accuracy, but the speed needs to be improved.

There are still some shortcomings in this paper. This paper uses the mechanical load test in the existing standards to simulate the static load of the photovoltaic support system. The theoretical calculation results and simulation simulation analysis and experiments still have some differences. The follow-up work should be continuously improved. The theoretical and simulation analysis results of the thesis, when the mechanical dynamic load test is performed with sufficient funds, it is necessary to more objectively and comprehensively verify and evaluate whether the design of the photovoltaic support system meets the requirements under various working conditions.

\section{References}

[1] Mohanty S , Subudhi B , Ray P K . A New MPPT Design Using Grey Wolf Optimization Technique for Photovoltaic System Under Partial Shading Conditions[J]. IEEE Transactions on Sustainable Energy, 2015, 7(1):1-8.

[2] Soon T K, Mekhilef S . A Fast-Converging MPPT Technique for Photovoltaic System Under FastVarying Solar Irradiation and Load Resistance[J]. IEEE Transactions on Industrial Informatics, 2015, 11(1):176-186.

[3] Kazem H A , Al-Waeli A H A, Chaichan M T , et al. Design, measurement and evaluation of photovoltaic pumping system for rural areas in Oman[J]. Environment Development and Sustainability, 2016, 19(3):123.

[4] La N , Lepore M, Bourdon Y, et al. Renewable Energy Desalination: Development of Photovoltaic Electrodialysis Desalination System[J]. Guangdong Chemical Industry, 2015, 3(1):: 13.

[5] Vorndran S D , Chrysler B , Wheelwright B, et al. Off-axis holographic lens spectrum-splitting photovoltaic system for direct and diffuse solar energy conversion[J]. Applied Optics, 2016, 55(27):75227529. 
[6] Chen X, Yang Y Y, Zhang Y J , et al. Influence of Illumination Probability of Photovoltaic System on Voltage of Power Distribution Networks[J]. Huanan Ligong Daxue Xuebao/Journal of South China University of Technology (Natural Science), 2015, 43(4):112-118.

[7] Azali S , Sheikhan M . Intelligent control of photovoltaic system using BPSO-GSA-optimized neural network and fuzzy-based PID for maximum power point tracking[J]. Applied Intelligence, 2016, 44(1):88110.

[8] Dhanapal S, Anita R . Voltage and Frequency Control of Stand Alone Self-Excited Induction Generator Using Photovoltaic System Based STATCOM[J]. Journal of Circuits, Systems, and Computers, 2016, 25(04):783-790.

[9] Guo X . A Novel CH5 Inverter for Single-Phase Transformerless Photovoltaic System Applications[J]. IEEE Transactions on Circuits \& Systems II Express Briefs, 2017, 64(10):1197-1201.

[10] Khoo B , Wee C C , Mariappan M, et al. A Hybrid Artificial Intelligence Algorithm to Determine the Speed and Position in Multi Operation Mode Sensorless Brushed D.C. Motor[J]. Advanced Science Letters, 2017, 23(11):11374-11377.

[11] Price S , Flach P A . Computational support for academic peer review: A perspective from artificial intelligence[J]. Communications of the ACM, 2017, 60(3):70-79.

[12] Kuppusamy P G . An Artificial Intelligence Formulation and the Investigation of Glaucoma in Color Fundus Images by Using BAT Algorithm[J]. Journal of Computational \& Theoretical Nanoscience, 2017, 14(4):1-5.

[13] Cath C , Wachter S , Mittelstadt B , et al. Artificial Intelligence and the 'Good Society': the US, EU, and UK approach[J]. Science and Engineering Ethics, 2017, 24(7625):1-24.

[14] Jiang F , Jiang Y, Zhi H, et al. Artificial intelligence in healthcare: Past, present and future[J]. Stroke \& Vascular Neurology, 2017, 2(4):230.

[15] Citakoglu H . Comparison of artificial intelligence techniques via empirical equations for prediction of solar radiation[J]. Computers and Electronics in Agriculture, 2015, 118(2015):28-37.

[16] Ashrafian H, Darzi A , Athanasiou T . A novel modification of the Turing test for artificial intelligence and robotics in healthcare[J]. International Journal of Medical Robotics and Computer Assisted Surgery, 2015, 11(1):38-43.

[17] Kaplan J . Artificial intelligence: think again[J]. Communications of the ACM, 2016, 60(1):36-38.

[18] Johnson K W , Soto J T , Glicksberg B S , et al. Artificial Intelligence in Cardiology[J]. Journal of the American College of Cardiology, 2018, 71(23):2668-2679.

[19] Suresh L P , Dash S S, Panigrahi B K . Artificial Intelligence and Evolutionary Algorithms in Engineering Systems: Volume 1[J]. Advances in Intelligent Systems \& Computing, 2015, 325(10):643-648.

[20] Ashrafian H . AIonAI: A Humanitarian Law of Artificial Intelligence and Robotics[J]. Science \& Engineering Ethics, 2015, 21(1):29-40. 
[21] Lee E J , Kim Y H , Kim N , et al. Deep into the Brain: Artificial Intelligence in Stroke Imaging[J]. Journal of Stroke, 2017, 19(3):277-285.

[22] Yeung S, Downing N L, Fei-Fei L, et al. Bedside Computer Vision - Moving Artificial Intelligence from Driver Assistance to Patient Safety[J]. New England Journal of Medicine, 2018, 378(14):1271-1273.

[23] Dignum V . Ethics in artificial intelligence: introduction to the special issue[J]. Ethics \& Information Technology, 2018, 20(1):1-3.

[24] Sernani P , Claudi A , Dragoni A F . Combining Artificial Intelligence and NetMedicine for Ambient Assisted Living: A Distributed BDI-based Expert System[J]. International Journal of E-Health and Medical Communications, 2015, 6(4):62-76.

[25] Lu X , Fei J. Velocity Tracking Control of Wheeled Mobile Robots by Iterative Learning Control[J]. International Journal of Advanced Robotic Systems, 2016, 13(3):1.

[26] Samir Lemeš,, Damir Štrbac,, Cabaravdic M. Using Industrial Robots to Manipulate the Measured Object in CMM[J]. International Journal of Advanced Robotic Systems, 2013, 10(281):1-9.

[27] Nair B B , Mohandas V P . Artificial intelligence applications in financial forecasting-a survey and some empirical results[J]. Intelligent Decision Technologies, 2015, 9(2):99-140.

[28] Bartsch G , Mitra A P , Mitra S A, et al. Use of Artificial Intelligence and Machine Learning Algorithms with Gene Expression Profiling to Predict Recurrent Nonmuscle Invasive Urothelial Carcinoma of the Bladder[J]. Journal of Urology, 2016, 195(2):493-498.

[29] Das S , Dey A , Pal A, et al. Applications of Artificial Intelligence in Machine Learning: Review and Prospect[J]. International Journal of Computer Applications, 2015, 115(9):31-41.

[30] Jeganathan J , Knio Z , Amador Y, et al. Artificial Intelligence in Mitral Valve Analysis[J]. Annals of Cardiac Anaesthesia, 2017, 20(2):129. 